\title{
ELECTROMAGNETIC DESIGN SEARCH AND OPTIMISATION OF PHOTONIC BANDGAP DEVICES ON DISTRIBUTED COMPUTATIONAL RESOURCES
}

\section{A6: Optimisation / C8: Photonic Bandgap Structures}

\author{
M Molinari ${ }^{1}$, K S Thomas ${ }^{2}$, S J Cox ${ }^{1}$ \\ ${ }^{1}$ School of Engineering Sciences \\ ${ }^{2}$ School of Electronics and Computer Science \\ University of Southampton, SO17 1BJ, UK \\ \{m.molinari@soton.ac.uk,kst@ecs.soton.ac.uk, sjc@soton.ac.uk\}
}

\begin{abstract}
Photonic crystals are devices with periodically modulated dielectric constant, designed to exhibit band gaps in a frequency spectrum in which electromagnetic waves cannot propagate.

Tuning the properties of these structures to achieve precise band gaps before fabrication is of high interest to photonic crystal manufacturers.

In this paper, we present the process of finding and optimising a photonic crystal design using a highthroughput Condor-based compute cluster and transparent database technology for easy storage, retrieval and re-use of the created designs. We also demonstrate how a band gap diagram can easily be obtained on a compute cluster when using the developed user interface technology. The optimisation process can easily be adapted to other problem areas.
\end{abstract}

\section{INTRODUCTION}

Photonic band gap (PBG) materials are periodic dielectric structures comparable to crystals that exhibit a photonic band gap analogous to the electronic band gap present in semiconductors. Electromagnetic waves in the frequency range of this band gap are completely excluded from being transmitted through the crystal. This effect leads to a range of applications of Photonic Crystals (PCs) including their use in high-speed optical communication networks, narrow bandwidth optical filters, and highly efficient narrow band tuneable lasers. PCs also offer enormous potential in the production of purely optical circuits.

The properties of this band gap depend highly on the underlying geometry of the dielectric structure. As the fabrication and experimental evaluation of these crystals is an expensive and time-consuming process, manufacturers try to simulate and tune the properties of PCs to specific application requirements in computational simulations.

An optimal design can be achieved by performing a parametric design search to get an overview of potential candidate designs before a localised optimisation is performed. These steps involve the repeated solution of an Eigenvalue problem

\section{APPLICATION EXAMPLE}

We consider the problem of optimising a typical Photonic Crystal design for a specific filter application where a band gap in the optical frequency range 600$700 \mathrm{~nm}$ is sought. The initial geometric design is a square lattice of cylindrical rods.

Parametric Design. The variable parameters we will consider in this sample problem are the rod radius, $R$, and the offset of alternating rows, $O$, with respect to the first row (see figure 1).

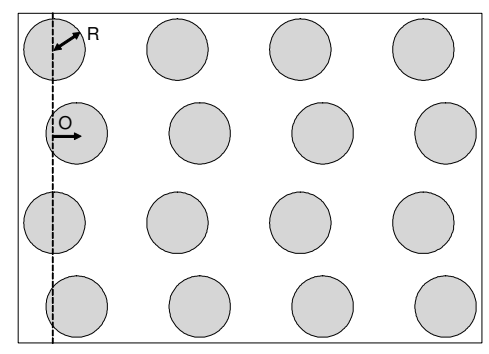

Figure 1: Parameterised geometry of a typical Photonic Crystal structure. Shown are the two sample variable parameters, radius $R$ and offset $O$.

This set-up corresponds to a two-dimensional parametric design search in the space $\{(O, R) \in[0,1] \times[0,0.5]\}$. To obtain an estimate of how the band gap depends on the design parameters, we will first carry out a LP-Tau based automatic parametric design sweep. The designs returned by this search contain valuable information for different applications and we thus retain the steps and results of the search process in a database for future reference and retrieval.

Numerical Solver. There are currently a number of solvers which give us the density-of-states or transmission spectrum of these designs. These include a unitcell based finite element algorithm (1) and a finite difference time domain solver as well as an enhanced 3D Vector finite element research code. The discretisation of the crystal geometry with finite elements, is performed from the geometry definition using publicly available meshing software (Easymesh, Geompack, Netgen) to produce a unit cell with matching elements at the periodic boundaries. This 
finite element mesh of a unit cell then enables us to use a fast finite element-based Eigenvalue solver developed at the University of Southampton. Alternatively, to obtain the corresponding optical transmission spectrum as indicated in figure 2, a FDTD algorithm can be employed on a PC structure of finite extend.

Objective Function Band Gap Extraction. In order to extract the band gap from the transmission spectrum, we post-process the eigenvalue spectrum obtained according to the application specification. Of interest are the mid-point and width of a band gap, and we define an objective function $(\Psi)$ through upper $(u)$ and lower $(l)$ limits on the transmission curve $(f)$ as shown in figure 2:

$$
\Psi=\sum_{i}\left(u_{i}-f_{i}\right)^{2}+\sum_{i}\left(f_{i}-l_{i}\right)^{2}-\sum_{i}\left(l_{i}^{2}+u_{i}^{2}\right)
$$

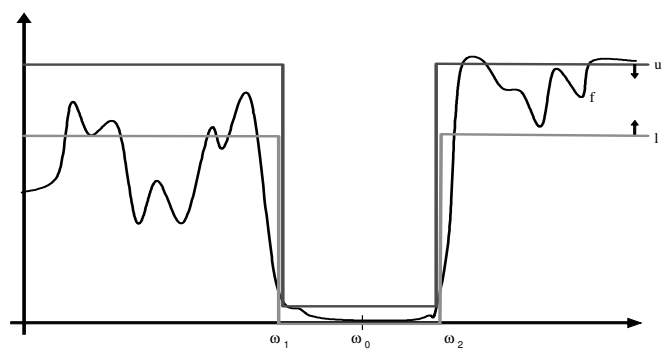

Figure 2: Construction of objective function through application of upper (u) and lower (1) limit constraints on a sample transmission spectrum (f).

The extracted band gap is characteristic for the design under investigation and varies according to the starting geometry. To find an optimal band gap for our application, we need to first find a design which exhibits a band gap close to the target. We then iteratively refine this design retrieved from the database with a gradientbased solver which converges to a local minimum of the objective function in the pre-selected region.

Computation. The large number of computations required for an initial design search can be handled efficiently by a cluster of workstations connected and managed through Condor High Throughput Computing software (2). Our local test-pool of Condor-enabled compute resources comprises of about 20 desktop stations, which are used in parallel and thus reduce computing time by a factor of approximately 20 .

As part of the GEM project (3), we have developed a software interface which allows engineers to transparently manage the submission, monitoring and execution of the design search and optimisation process on this cluster. The integrated database features (4) allow an easy process flow creation as well as the transparent archiving, searching and retrieval of existing designs and compute runs.

Visualisation. The results obtained from the band gap extraction can be interpolated to give an image which offers an engineer valuable indications of where useful band gaps can be found. Thus an efficient pre-selection of the range of design to be investigated can be carried out on the basis of existing design calculations. If this is constraint to the parametric sweep of a single parameter, we obtain, for example, a band gap map as shown in figure 3 , with optical frequency on the horizontal axis and sample radius variations on the vertical axis.

\section{CONCLUSIONS \& FURTHER WORK}

Initial results show that a design search can be carried out efficiently when distributed compute resources such as a Condor-based cluster of workstations, are available. The band gap values extracted from the design points have been used as starting points for design optimisation and have also given a good indication of the device's characteristics before fabrication stage is reached.

With the developed software interface, the process of assembling, submitting, and executing this design search and the optimisation process, becomes very easy and straightforward.

Our current progress shows that the building of and access to repositories of designs and algorithms held in databases is an important tool which facilitates the work in engineering design search and optimisation.

We have used the tool successfully for research into characteristics of photonic crystals for a specific filter application.

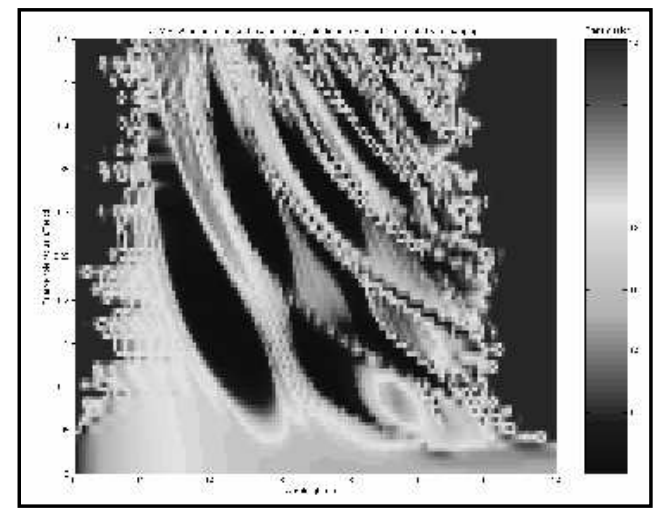

Figure 3: Typical band gap diagram for dielectric rods $(\varepsilon=8.9)$ in air. The abscissa shows optical frequencies range/wavelength, the ordinate represents variation in rod radius. Each row has been obtained on a different compute resource.

\section{ACKNOWLEDGEMENTS}

We would like to thank Dr Ben Hiett, Dr Duan Beckett, and Dr Jacek Generowicz for their work on PCs as well as the Geodise team for their work on EDSO.

\section{REFERENCES}

(1) Beckett D.H., Cox S.J., Generowicz J.M., Hiett B.P., Molinari M., Thomas K.S.; CEM 2002

(2) The Condor Project, http://www.cs.wisc.edu/condor/

(3) The GEM Project, http://www.soton.ac.uk/ gridem

(4) Wason, J.L., Molinari M., Jiao Z., Cox, S.J., 2003 Euro-Par 2003 Parallel Processing, 413-416 